\title{
Temperature Control of Solid Oxide Fuel Cell System with Coefficient Diagram Method
}

\author{
Chananchai WUTTHITHANYAWAT ${ }^{1,{ }^{*}}$ and Nawadee SRISIRIWAT ${ }^{2}$ \\ ${ }^{1}$ Department of Instrumentation and Control Engineering, Faculty of Engineering, \\ ${ }^{2}$ Department of Petrochemicals and Environmental Management, Faculty of Engineering, \\ Pathumwan Institute of Technology, Bangkok, Thailand. \\ *chananchai.wut@pit.ac.th
}

Keywords: Coefficient Diagram Method, Multiloop control, Solid oxide fuel cell.

\begin{abstract}
In this work, we address a coefficient diagram method (CDM) for designing temperature control available to an energy integrated solid oxide fuel cell (SOFC) system that the high temperature of SOFC exhaust gas can be a heat source for all heat exchangers in SOFC system. The SOFC system represents the multi-loop control system in which the key controlled variables are the SOFC, steam reformer and heat exchanger temperatures. For temperature control design, an air flow rate entering SOFC and a valve position of SOFC hot stream splitter are identified as the manipulated variables to regulate the main controlled variables of a SOFC temperature and a steam reformer temperature, respectively. By using the CDM, the stability and robustness for regulating the SOFC system temperatures were analyzed to offer the suitable control parameters and the simulation results showed that the designed temperature control of SOFC system provides a good performance to control the controlled variables and reject the disturbance.
\end{abstract}

\section{Introduction}

High temperature solid oxide fuel cell (SOFCs), integrated with fuel reforming technologies, can be promised to be very efficient for converting chemical energy into electricity. It also provides high system efficiency, low environmental impact and flexibility of usable fuel types. Another advantage of the SOFC is the use of high temperature exhaust gas from SOFC outlet as the usable heat that makes it suitable to integrate heat with fuel processor and all heat exchangers in the SOFC system as proposed in a previous work of modeling and simulation of an energy integrated SOFC system when ethanol solution reformed to hydrogen-rich gas was chosen [1]. Moreover, the energy integrated SOFC and heat recovery gas turbine can generate the additional electricity using for driving a liquid pump for ethanol solution and an air compressor in the SOFC system. Furthermore, the energy integration is achieved by designing a network of heat exchanger [2].

In our previous work [1], the modeling and simulation of SOFC system has been performed to characterize the dynamic behavior of the heat integration of SOFC system. The simulation results indicated that for the SOFC control loop, the air flow rate was designed as the manipulated variable $(\mathrm{MV})$ and the temperature of SOFC was designed as the controlled variable (CV) while for the steam reformer control loop, the valve position of high temperature SOFC exhaust gas splitter was considered as the MV when the temperatures of steam reformer was designed as the CV.

There are many control design methods applying for the SOFC system and Huang et al. [3] also presented excellent overviews on perspective of dynamic modeling and control for the SOFC. In case of the energy integration of SOFC systems, various integration strategies for SOFC energy systems proposed by Zhang et al. [4]. Georgis et al. [5-6] carried out the design and operation of an energy integrated SOFC system with an external methane steam reformer and applied a linear multi-loop control strategy developed for the entire integrated system [5] and a nonlinear model based controller derived for the control of fuel cell [6]. Moreover, many researches [7-10] investigated the control strategies of the combination of SOFC and gas turbine system that main control objective is to 
maintain SOFC stack temperature, fuel flow rate and fuel utilization. However, most of researches used methane reforming to produce the hydrogen-rich gas as fuel for SOFC system but, nevertheless, the literature on the modeling and control of the integrated SOFC system with an ethanol steam reformer is limited.

In this paper, a new challenge is made up to design a PI controller for an energy integrated SOFC system using coefficient diagram method (CDM). The main features of CDM are modification of the polynomial expressions for the plant and the controller, nonexistence or existence of very small overshoot in the closed loop response, obtaining the characteristic polynomial of the closed loop system efficiently by taking a good balance of stability, time response and robustness of the control system with uncertainly in the plant parameters [11]. Consequently, many control systems have been designed successfully using CDM [11-13]. As the strength of CDM lies in that the simplest and robust controller under practical limitations can be found for any plant [12], this work also investigates the multi-loop control design for the energy integrated SOFC system using CDM.

\section{An Energy Integrated SOFC System}

The high operating temperature of SOFC offers the possibility of both internal and external steam reformer (SR) from ethanol to produce the hydrogen-rich gas as the fuel of SOFC. Fig. 1 shows the basic design of heat exchanger network for an energy integrated SOFC system based on the pinch technology. The details of the energy integrated SOFC system were explained in the previous work [2].

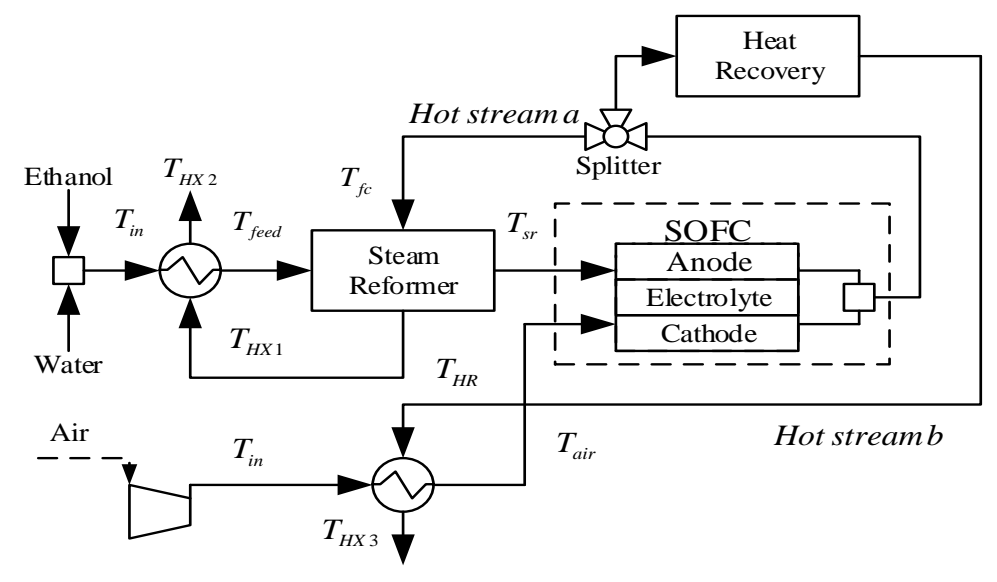

Figure 1. Basic structure design of an energy integrated SOFC system.

\section{CDM Procedure}

One of algebraic approaches of a control design procedure based on polynomial expressions is the coefficient diagram method (CDM) introduced by Manabe [14] that the numerator and denominator of the transfer function are considered independently from each other so better results can be achieved against pole-zero cancellation [15]. The prominent point of CDM is its simplicity and robust controller for several industry processes under practical limitation and various control systems have been designed successfully by using CDM [11]. A good control system can be designed by using efficient CDM control tool. It is easy to realize a controller under the conditions of stability, time domain performance and robustness [16]. The close relations between these conditions and coefficients of the characteristic polynomials can be simply determined. This means that CDM is effective for not only control system design but also controller tuning [12, 17]. In CDM, designed specification parameters are equivalent time constant $(\tau)$, stability index $\left(\gamma_{i}\right)$ and stability limits $\left(\gamma_{i}^{*}\right)$.

The basic block diagram of the CDM control system is demonstrated in Fig. 2, where $N(s)$ and $D(s)$ are numerator and denominator polynomial transfer functions of the plant. $A(s)$ is the forward 
denominator polynomial, while $F(s)$ and $B(s)$ are considered as the reference numerator and the feedback numerator polynomials in CDM controller transfer functions, respectively. As the controller transfer function has two numerators, it resembles to a 2DOF (Degree of Freedom) system structure. $A(s)$ and $B(s)$ are designed as to satisfy the required transient behavior, while pre-filter $F(s)$ is determined as zero order polynomial and used to provide the steady-state gain [16]. The output of the CDM control system from Fig. 2 can be written as

$$
Y(s)=\frac{N(s) F(s)}{P(s)} R(s)+\frac{A(s) N(s)}{P(s)} D(s) .
$$

Where $P(s)$ is the characteristic polynomial of the close loop system that $P(s)$ can be defined by

$$
P(s)=A(s) D(s)+B(s) N(s)=\sum_{i=0}^{n} a_{i} s^{i} ; a_{i}>0 .
$$

The $A(s)$ and $B(s)$ polynomials in the CDM control structure are given by

$$
A(s)=\sum_{i=0}^{p} l_{i} s^{i} \text { and } B(s)=\sum_{i=0}^{q} k_{i} s^{i} ; p \geq q
$$

According to Manabe [14], the CDM design parameters, including equivalent time constant $(\tau)$, stability index $\left(\gamma_{i}\right)$ and stability limits $\left(\gamma_{i}^{*}\right)$ are defined as Eq. 4a, Eq. 4b and Eq. 4c, respectively [12, $18]$.

$$
\begin{aligned}
& \tau=\frac{a_{1}}{a_{0}} \\
& \gamma_{i}=\frac{a_{i}^{2}}{\left(a_{i-1} a_{i+1}\right)} ; i=1, \ldots, n-1 \\
& \gamma_{i}^{*}=\frac{1}{\gamma_{i+1}}+\frac{1}{\gamma_{i-1}} \quad ; \quad \gamma_{o}=\gamma_{n}=\infty
\end{aligned}
$$

The equivalent time constant is determined the time response speed. The stability index specifies the stability and the waveform of the time response. The variation of the stability index owing to plant parameters variation designates the robustness property $[12,14]$.

The key parameters, which are firstly chosen, for CDM control system are the equivalent time constant and the stability index. For the choice of the equivalent time constant, the desired settling time for the time response of the CDM control system is calculated before starting the design. The relation between the settling time and the equivalent time constant is considered according to the standard Manabe form [14]. If $t_{s}$ denotes the desired settling time, the relation express of the chosen $\tau$ can be given by

$$
\tau=\frac{t_{s}}{(2.5 \sim 3)}
$$

For the choice of the stability index, according to the Manabe form [14], the stability index is chosen as

$$
\gamma_{1}=2.5, \quad \gamma_{i}=2 ; i=2 \sim(n-1), \quad \gamma_{0}=\gamma_{n}=\infty .
$$

The standard values of stability index according to the Manabe form [14] can be used to design the controller if the following condition as presented in Eq. 7 is satisfied. 


$$
p_{k} / p_{k-1}>\tau /\left(\gamma_{n-1} \gamma_{n-2}, \ldots \gamma_{1}\right)
$$

Where $p_{k}$ and $p_{k-1}$ are the coefficients of the plant at $k^{\text {th }}$ and $(k-1)^{\text {th }}$ order, respectively. If the above condition is not satisfied, we can first increase $\gamma_{n-1}$ then $\gamma_{n-2}$ and so on, until Eq. 7 is satisfied. Using the designed parameters $\tau$ and $\gamma_{i}$, a target characteristic polynomial, $P_{t \text { arg } e t}(s)$ is obtained as

$$
P_{t \arg e t}(s)=a_{0}\left[\left\{\sum_{i=2}^{n}\left(\prod_{j=1}^{i-1} \frac{1}{\gamma_{i-j}^{j}}\right)(\tau s)^{i}\right\}+\tau s+1\right] .
$$

By equating the two characteristic polynomials in Eq. 2 and Eq. 8, a Diophantine equation (Eq. 9) is obtained. The controller parameters $k_{i}$ and $l_{i}$ are computed by solving this equation easily.

$$
A(s) D(s)+B(s) N(s)=P_{t \arg e t}(s)
$$

From the CDM standard block diagram, it can be rearranged in a structure of 2 DOF [19] as illustrated in Fig. 3 where the controller $G_{c}(s)$ and the pre-filter $G_{f}(s)$ are defined as Eq. 10 and Eq. 11, respectively.

$$
\begin{aligned}
& G_{c}(s)=\frac{B(s)}{A(s)} \\
& G_{f}(s)=\frac{F(s)}{B(s)}
\end{aligned}
$$

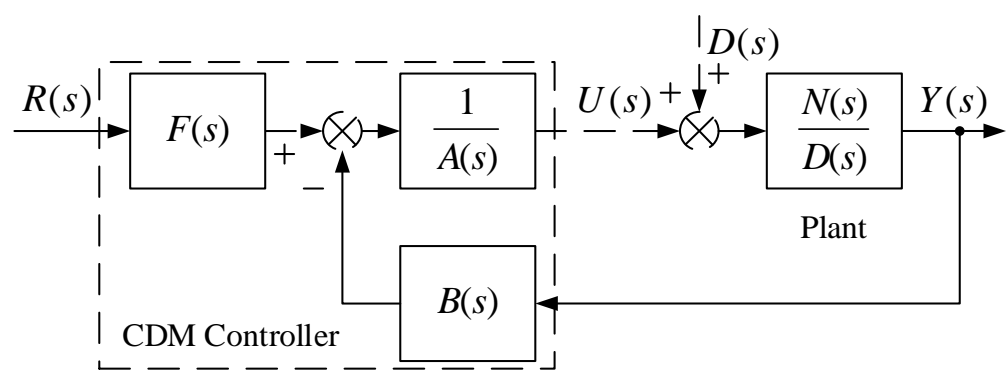

Figure 2. A block diagram of CDM control system.

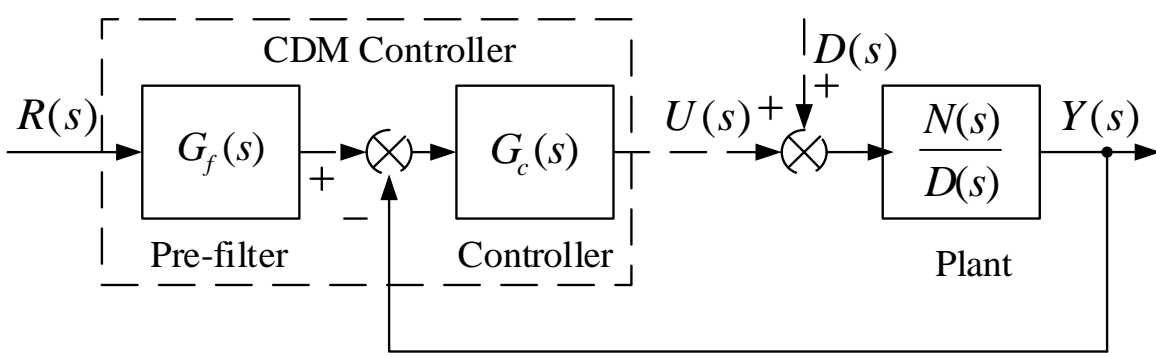

Figure 3. A rearranged block diagram of CDM control system.

\section{Control Design using CDM}

The control objectives of the energy integrated SOFC system are considered for the entire integrated SOFC system as follows:

Fuel cell temperature control: The SOFC system is assumed that all the gases inside the SOFC stack are perfectly mixed; thus the temperature of exhaust gas from the SOFC can be consider as same 
as the operating temperature. The SOFC temperature can be controlled by manipulating the air flow rate of the system [1]. The set point temperature is $1200 \mathrm{~K}$. Based on the unsteady-state energy balance equations in the previous study of modeling and simulation of the energy integrated SOFC system [1], the transfer function of SOFC system can be written as

$$
G_{\text {SOFC }}=\frac{T_{f c}(s)}{N_{\text {air }}(s)}=\frac{1.161}{73.73 s+1} .
$$

Where $T_{f c}$ is the SOFC temperature and $N_{a i r}$ is the air molar flow rate. Nominal parameters and operating points of this SOFC system are presented in previous work [1].

According to CDM design procedure as mentioned in previous section, by using Eq. 5, the desired settling time $\left(t_{s}\right)$ of $200 \mathrm{sec}$ is defined; thus the equivalent time constant $(\tau)$ can be calculated as 80 sec. When the stability index was varied as $2.5,3,4,5$ and 10 , the controller parameters of CDM-PI control can be determined as shown in Table 1.

Steam reformer temperature control: The temperature of steam reformer mainly affects both the composition of gas product influencing the amount of electrical power generation of SOFC and the temperature of SOFC stack. The steam reformer temperature can be controlled by adjusting the valve position of hot stream splitter [1]. The set point temperature is $973 \mathrm{~K}$ to produce the required gas composition fed into anode side of SOFC. The transfer function of steam reformer unit (HX1) is

$$
G_{H X 1}=\frac{T_{s r}(s)}{v p_{1}(s)}=\frac{0.01065 s+0.01728}{128.9 s^{2}+212.4 s+1} .
$$

where $T_{s r}$ is the steam reformer temperature and $v p_{1}$ is the valve position calculated as the mass flowrate in the hot stream $a$ divided by the total mass flowrate of the SOFC's outlet stream.

The transfer function of HX1 in Eq. 13 can represent to an estimated model of the first order system as shown in Eq. 14, that is

$$
G_{E s t . H X 1}=\frac{0.01728}{250 s+1} \text {. }
$$

In this steam reformer control loop, the desired settling time $\left(t_{s}\right)$ is also specified at $200 \mathrm{sec}$ so the equivalent time constant $(\tau)$ of $80 \mathrm{sec}$ can be also determined. By varying the stability index to be 2.5 , $3,4,5$ and 10, the controller parameters of CDM-PI control can be determined as shown in Table 2.

Table 1. Controller Parameters of CDM-PI for Different Values of Stability Index of SOFC Temperature Control.

\begin{tabular}{|c|c|c|c|}
\hline Stability index, $\gamma_{1}$ & $\mathbf{K}_{\mathbf{p}}$ & $\mathbf{K}_{\mathbf{i}}$ & $\tau_{i}$ \\
\hline 2.5 & 1.1232 & 0.0248 & 45.2826 \\
\hline 3 & 1.5200 & 0.0298 & 51.0688 \\
\hline 4 & 2.3137 & 0.0397 & 58.3016 \\
\hline 5 & 3.1074 & 0.0496 & 62.6413 \\
\hline 10 & 7.0759 & 0.0992 & 71.3206 \\
\hline
\end{tabular}

Table 2. Parameters of CDM-PI for Different Values of Stability Index of Steam Reforming Temperature Control.

\begin{tabular}{|c|c|c|c|}
\hline Stability index, $\gamma_{1}$ & $\mathbf{K}_{\mathbf{p}}$ & $\mathbf{K}_{\mathbf{i}}$ & $\tau_{i}$ \\
\hline 2.5 & 394.1963 & 5.6507 & 69.7600 \\
\hline 3 & 484.6083 & 6.7809 & 71.4667 \\
\hline 4 & 665.4322 & 9.0412 & 73.6000 \\
\hline 5 & 846.2562 & 11.3015 & 74.8800 \\
\hline 10 & $1,750.4000$ & 22.6030 & 77.4400 \\
\hline
\end{tabular}




\section{Simulation Results}

The testing of performance of CDM-PI controller system, when the step response of SOFC temperature was changed by $+5 \%$, is shown in Fig. 4 . The effects of stability index varying at $2.5,3,4$, 5 and 10 on the response of SOFC temperature and control signal of air valve are illustrated in Figs. 4(a) and 4(b), respectively. An increase of the stability index increases the settling time but decreases the overshoot of control system when the higher stability index, the higher maximum control signal as shown in Table 3. The stability index also affects the SR temperature and control signal of hot stream valve as illustrated in Figs. 4(c) and 4(d), respectively. The change of SOFC temperature influences the SR temperature when the stability index of SR control loop increases, the peak of SR temperature decreases and it can be found that the control system can rapidly regulate the SR temperature to the nominal operating point.

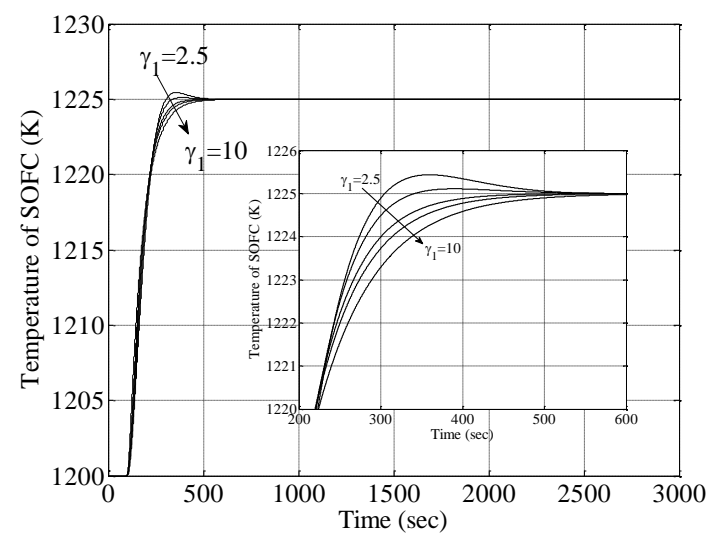

(a)

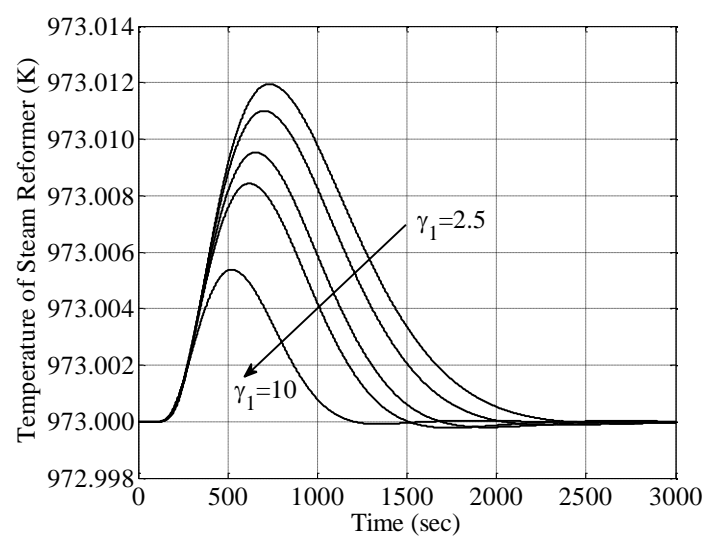

(c)

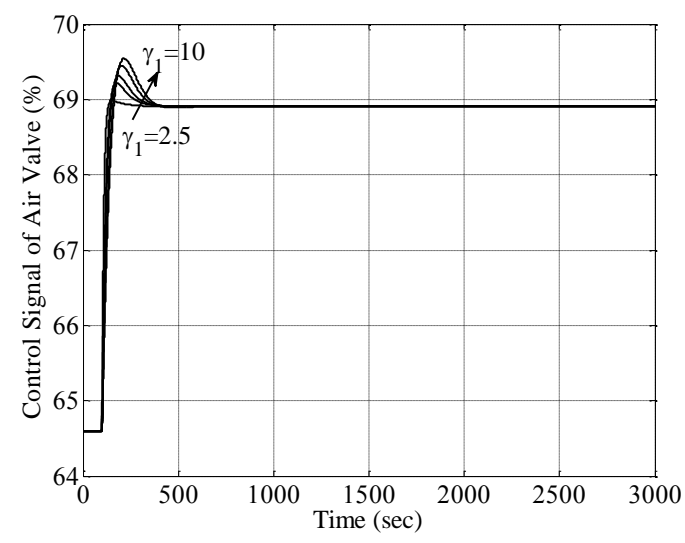

(b)

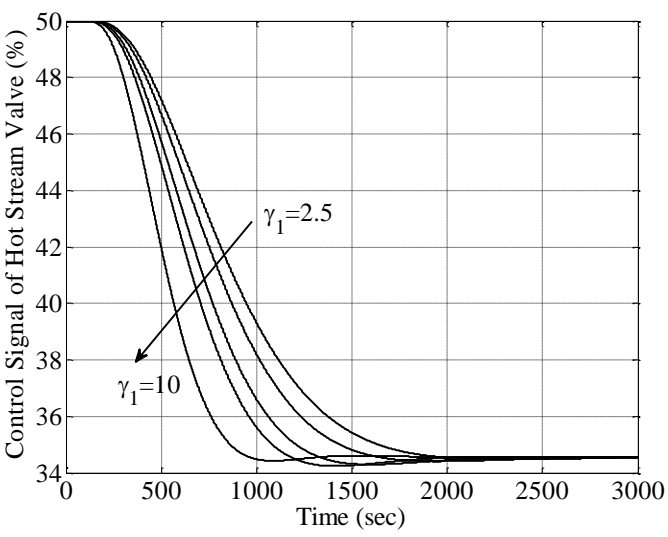

(d)

Figure 4. Step response of SOFC system loop (a) Response of SOFC temperature, (b) Control signal of air valve, (c) Response of steam reformer temperature and (d) Control signal of hot stream valve.

Table 3. Comparison Performance Values of Time Response Curves in Figs. 4(a) and 4(b).

\begin{tabular}{|c|c|c|c|}
\hline Stability index, $\gamma_{1}$ & $P_{o}(\%)$ & $t_{s}(\mathrm{sec})$ & $u_{\max }(\%)$ \\
\hline 2.5 & 2 & 187 & 68.98 \\
\hline 3 & 0.4 & 201 & 69.22 \\
\hline 4 & 0 & 233.5 & 69.31 \\
\hline 5 & 0 & 254 & 69.45 \\
\hline 10 & 0 & 287.5 & 69.55 \\
\hline
\end{tabular}

In order to investigate the disturbance rejection, the step change of air temperature was increased $10 \%$ from the nominal operating point as shown in Fig. 5. A rapid change of air temperature increases SOFC temperature (see Fig. 5(a)) and affects the SR temperature (see Fig. 5(c)); however, an increase of the stability index can decrease the change of SOFC and SR temperatures and they can be adjusted to be the nominal operating point rapidly. 
The increase of stability index can decrease the overshoot but increase the settling time that means the control system can robust the disturbance of the energy integrated SOFC plant and can reject the disturbance rapidly.

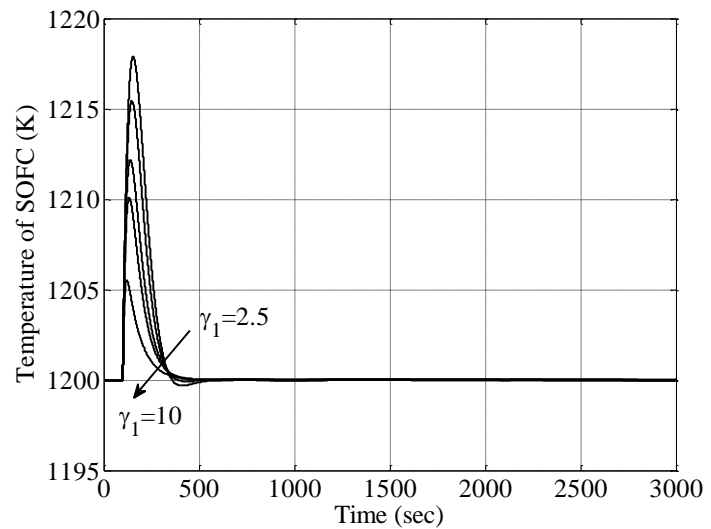

(a)

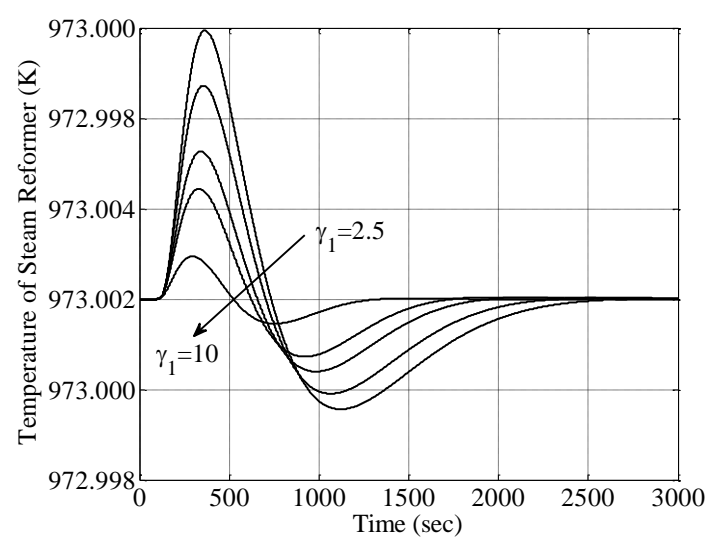

(c)

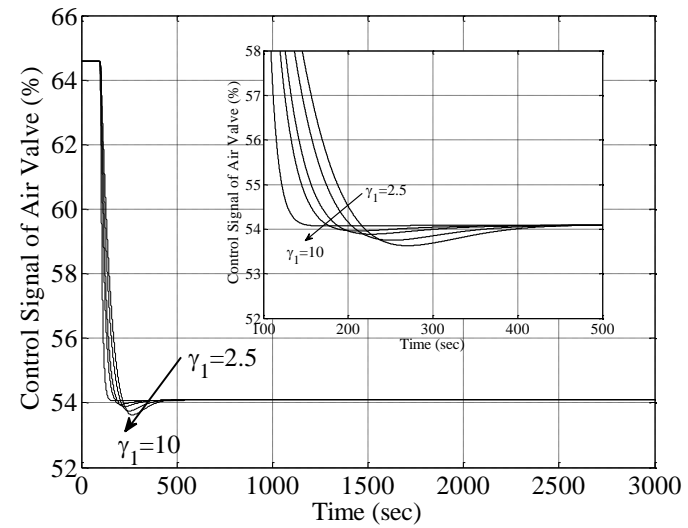

(b)

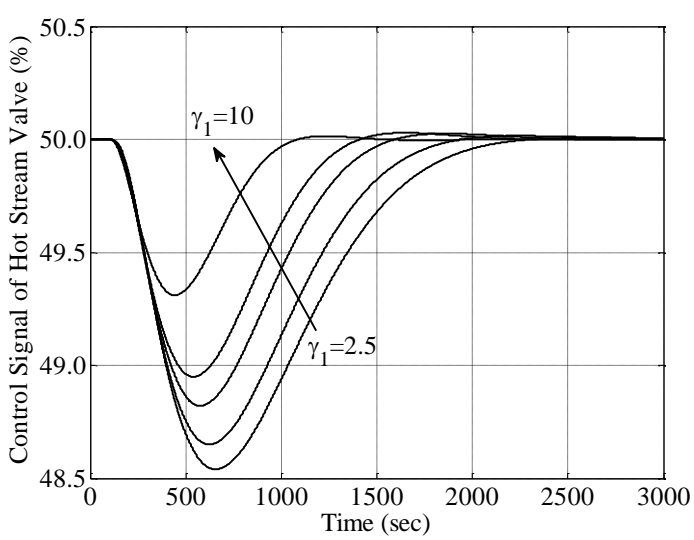

(d)

Figure 5. Closed loop response of air inlet temperature disturbance (a) Response of SOFC temperature, (b) Control signal of air valve, (c) Response of steam reformer and (d) Control signal of hot stream valve.

\section{Conclusion}

In this paper, the control system design with CDM for the temperature control of SOFC system was presented to maintain the SOFC and SR temperatures. The control parameters were determined by $\mathrm{CDM}$ based on PI controller. The PI controller parameters were estimated by defining the stability index and equivalent time constant. The results showed that overshoot decreases but the settling time increases when the stability index increases. Moreover, this designed control system can reject the disturbance and provide the good performance and the robustness with increasing stability index.

\section{References}

[1] C. Wutthithanyawat, N. Srisiriwat, An Energy Integrated Solid Oxide Fuel Cell System: Modeling and Simulation, Applied Mechanics and Materials, 446-447 (2014) 777-783.

[2] N. Srisiriwat, C. Wutthithanyawat, Heat Integration of Solid Oxide Fuel Cell System, Applied Mechanics and Materials, 541-542 (2014) 922-926.

[3] B. Huang, Y. Qi, A.M. Murshed, Solid oxide fuel cell: Perspective of dynamic modeling and control, J. Process Control, 21 (2011) 1426-1437. 
[4] X. Zhang, S. Chan, G. Li, H. Ho, J. Li, Z. Feng, A review of integration strategies for solid oxide fuel cells, J. Power Sources, 195 (2010) 685-702.

[5] D. Georgis, S.S. Jogwar, A.S. Almansoori, P. Daoutidis, Design and control of energy integrated SOFC systems for in situ hydrogen production and power generation, Comput. Chem. Eng. 35 (2011) 1691-1704.

[6] D. Georgis, S.S. Jogwar, A.S. Almansoori, P. Daoutidis, Control of an Energy Integrated Solid Oxide Fuel Cell System, Proc. 2011 American Control Conference, (2011) 1518-1523.

[7] W. Jiang, R. Fang, J. Khan and R. Dougal, Control Strategies for Start-Up and Part-Load Operation of Solid Oxide Fuel Cell/Gas Turbine Hybrid System, Journal of Fuel Cell Science and Technology, 7 (2010) 1-9.

[8] Christoph Stillera, Bjørn Thoruda, Olav Bollanda, Rambabu Kandepub and Lars Imslandb, Control strategy for a solid oxide fuel cell and gas turbine hybrid system, J. Power Sources 158 (2006) 303-315.

[9] T. Kaneko, J. Brouwer, G.S. Samuelsen, Power and temperature control of fluctuating biomass gas fueled solid oxide fuel cell and micro gas turbine hybrid system, J. Power Sources 160 (2006), 316-325.

[10] Jiqing He, Peilin Zhou, David Clelland, The development of control strategy for solid oxide fuel celland micro gas turbine hybrid power system in ship application, J Mar Sci Technol 19 (2014) 462-469.

[11]Rinu Raj R. R, L. D Vijay Anand, Design and Implementation of a CDM-PI Controller for a Spherical Tank Level System, International Journal on Theroretical and Applied Research in Mechanical Engineering, 2 (2013) 49-52.

[12] M. Koksal, S.E. Hamamci, Robust Temperature Control of MSF Desalination Plants with Coefficient Diagram Method, Proc. 2003 IEEE Conference on Control Applications, (2003) 1437-1442.

[13] N. Kanagasabai, N. Jaya, Design of Multiloop Controller for Three Tank Process Using CDM Techniques, International Journal on Soft Computing, 5 (2014) 11-20.

[14] S. Manabe, Coefficient Diagram Method, Proc. of the $14^{\text {th }}$ IFAC Symposium on Automatic Control in Aerospace, Seoul, (1998) 199-210.

[15] S.E. Hamamci, I. Kaya, D.P. Atherton, Smith Predictor Design by CDM, Proc. 2001 European Control Conference (ECC), (2001) 2364-2369.

[16] S. Somasundaram, P.K. Bhaba, Control of Bio-Reactor Processes using a New CDM PI-P Control Strategy, Journal of Engineering Science and Technology, 5 (2010) 213-222.

[17] S. Somasundaram, P.K. Bhaba, A New CDM PI - PD Control strategy for unstable processes, International Journal of Engineering Simulation, 10 (2009) 19-24.

[18] S. Manabe, "Imporatance of Coefficient Diagram in Polynomial Method," Proc. the $42^{\text {nd }}$ IEEE Conference on Decision and Control, (2003) 3489-3494.

[19] T. Benjanarasuth, J. Ngamwiwit, N. Komine, "Simple two-degree of freedom PID controllers tuning table based on CDM", Int. Conf. on Control Automation and Systems, (2004) 256-261. 